Orthopäde

1997 $26: 407 @$ @ Springer-Verlag 1997

Editorial

\title{
Neue Techniken in der Unfallchirurgie
}

auch in der Wirbelsäulenchirurgie zu beobachten ist: Semiperkutane, kanülierte Systeme zur transartikulären Verschraubung $C_{1} / 2$ und transthorakale/ transabdominale endoskopiegestütze Techniken sind einige der neuen, gegenwärtig evaluierten Wege.

Die Minimierung des iatrogenen Weichteilschadens ist neben Präzisionsverbesserung und Reduktion von Röntgenstrahlen auflange Sicht ebenfalls Ziel der computerassistierten Wirbelsäulenchirurgie. U. Berlemann et al. aus der Forschergruppe um L. Nolte in Bern demonstrieren aktuelle Entwicklungen und das enorme Potential dieser neuen Form chirurgischer Technik.

Für resorbierbare, kompressionsfeste Knochenzemente zur Abstützung posttraumatischer, spongiöser Impressionszonen besteht in Zeiten eines zunehmenden geriatrischen Krankenguts steigender Bedarf. L. Claes et al. beschreiben Grundlagen und Entwicklung dieser Stoffgruppe, die gerade in den letzten Jahren vermehrt im Mittelpunkt des Interesses von Orthopäden und Unfallchirurgen stand.

Dem Einsatz von resorbierbaren Implantaten widmet sich der Beitrag von Rehm et al. Er setzt sich vor allem mit Polylactidstiften auseinander und präsentiert die Ergebnisse einer breit angelegten, prospektiv randomisierten multizentrischen Studie mit 183 Radiusköpfchenfrakturen.

Weichteildefekte sind für Unfallchirurgen und Orthopäden stets ein Thema. In dem systematisch angelegten Beitrag von A. Berger wird, basierend aufden Erfahrungen von über 1200 freien mikrovaskulären Gewebetransplantationen über Fortschritte, Möglichkeiten und Differentialindikation der verschiedensten Weichteildeckungstechniken berichtet.
Wegen der starken Abhängigkeit der Unfallchirurgie von bildgebenden Verfahren kommt der Kooperation mit der Radiologie eine herausragende Bedeutung zu. Schnellere und höher auflösende CT und MRT Untersuchungen, digitale Bildarchivierung, Subtraktionstechnologien und die Bereitstellung von Schnittstellen zur Computernavigation sind nur einige neue Entwicklungen, die $i m$ Beitrag von K. Wolf und K. Bohndorf präsentiert werden.

Wie in Zukunft in Unfallchirurgie und Orthopädie über Lösungsansätze nachgedacht werden kann und muß, wird im abschließenden Beitrag von T. Gehrich et al. deutlich. Er zeigt, daß die Manipulation von Vorgängen auf subzellulärer Ebene einschließlich der genetischen Informationen zur Verbesserung von Heilungsvorgängen bei Verletzungen des Binde- und Stützgewebes keine Utopie mehr ist.

Das vorliegende Heft soll nicht nur "Techniken" aufzeigen, sondern auch werten. Wertung setzt Erfahrung voraus - Erfahrung mit größeren Fallzahlen, die in der Regel für „neue Verfahren“ noch gar nicht vorliegen können.

Ein Themenheft wie dieses bewegt sich somit auf einem schmalen Grat. Lesen Sie selbst, ob den Autoren diese Gratwanderung gelungen ist.

C. Krettek und H. Tscherne

Die Herausgeber 